\title{
Target Signatures and Pose Estimation
}

\author{
Migdat I. Hodzic \\ American University in Bosnia and Herzegovina \\ International University of Sarajevo, FENS \\ Sarajevo, Bosnia \& Hercegovina \\ migdat.hodzic@aubih.edu \\ mhodzic@ius.edu.ba
}

\author{
Tarik Namas \\ Faculty of Engineering \& Natural Sciences, EE Program \\ International University of Sarajevo \\ Sarajevo, Bosnia \& Hercegovina \\ tnamas@ius.edu.ba
}

\section{ABSTRACT}

The paper discusses an important class of defense and commercial applications in the context of Ground Target (Object) Identification, Classification, and Tracking. The data base of target digital signatures is assembled and formed for a full spatial circle (360 degrees) analysis from such sources as High Resolution Radar and Synthetic Aperture Radar. These digital signatures are analized from which various spatial as well as frequency (wavelet) characteristics of the targets are formed and interpreted, in order to make good estimate of target pose angle. This angle is key for tracking maneouvering targets. Various statistical measures are obtained from digital signatures to assist in pose angle estimation. We also use certain geometrical considerations to determine an initial pose estimate which is the refined using a variety of correlation coefficients. Expected precision of pose estimate is within few degrees, i.e. within few neighboring target signatures. The paper presents several real life ground target signatures as well as several simulated signatures to illustrate our approach.

Keywords-Target Signatures, Target Data Base, Target Identification, Pose Angle Estimation, Statistics, Correlations

\section{INTRODUCTION}

Modern developments in the areas of various high resolution radar technologies, made the ground targets reflection signatures much richer and useful features of these targets can be identified. Successful target identification and tracking exploit these feature information to determine target type and its dynamics. In the process of automatic target recognition, the pose angle estimates are obtained as well, provided target data base contains full $360^{\circ}$ target signatures in small angle increments. Figure 1 shows typical pose angle (aspect and depression) geometry. The depression angle relates to sensor position and aspect angle is deduced from radar signatures. For ground targets, since their velocity vector is aligned most of the time with the body's longitudinal axis, the pose angles carry kinematic information that can be used to improve target tracking particularly during the target maneuvering periods [3-14].

In this paper we continue our previous and continuing work related to new methodology for improved automatic target identification as well as pose estimate, which can in turn improve target tracking and reduce target miss-association probability. In a typical airborne application (USA JSTAR is good example) [15-18], a ground target is detected from a distance, identified and classified using stored target data base, and then tracked after that. In this paper we expand our work reported in [1, 2], where we analyzed certain number of targets in spatial domain in particular. Section 2 in this paper reviews several targets signatures from USA public Moving and Stationary Target Acquisition and Recognition (MSTAR) program database [15-18]. The MSTAR data consists of SAR data in X-band, with $1 \times 1$ foot resolution, at $15^{\circ}$ depression angle. In addition to these real targets we generate a number of our own synthetic targets as a reference targets to test our pose estimation methodology. In summary, we average radar raw data along $\mathrm{X}$ and $\mathrm{Y}$ coordinates and test them individually for target identification purposes. Each target has 274 signatures at different pose angles, for a full circle, given in a form of a radar digital bit map. We form corresponding target data base with additional target features useful in aiding in real time target identification, as elaborated in Section 3. In particular we use target geometry as well as autocorrelation and cross correlation spatial characteristics to draw conclusions about target features which would place it in a certain precise place in the target data base. We analyze all 274 individual target signatures (total of $360^{\circ}$ ) and form a variety of statistical measures to associate with each signature. Another approach is to use second order statistics such as autocorrelation envelopes with minimum and maximum frequency information, which can be identified with certain geometric and spatial dimensions of the targets. We will also explore frequency (wavelet) aspect of the signature data in our future work. Note that in this case the "frequency" is an inverse of spatial rather than time coordinates. Besides standard first and second order statistics we also employ newly introduced statistical notion of Brownian Distance and the corresponding correlation index [19] which both measure independency of two random sequences. This is very powerful method and we employ it in target signature independence testing, for different targets and poses as well. Overall, the purpose of additional signature statistical analysis is to gain insight into key signature features which discriminate against other targets. The resulting expanded target data base has original raw radar data, as well as additional target features and statistics (Figure 4). This stored information is used in a subsequent real time target identification when its signature is compared against the data base in order to correctly identify the target with the lowest possible probability of wrong association. At the same time we also estimate pose in the process which in turn makes target tracking much easier and more precise. One notable historical ATR application is well known USA Air-force JSTAR platform [16-18] which was deployed in mid and late 1990s. In Bosnia and Herzegovina to enforce Dayton Peace Agreement (Kosovo as well). The platform was used for ATR and large arms collection confirmations. There is a current JSTAR modernization effort under way. Our methodology is applicable to other defense as well as commercial areas, such as traffic control. 


\section{TARGET SIGNATURE DATA BASE}

Basic target and sensor platform geometry is given in Figure 1. In [1] we used three signatures for analysis, and they are repeated here as a reference in Figures 2 (raw continuous data) and Figure 3 (16 digitized signature values). In [2] we expanded our spatial analysis to many more targets, digitization was at the

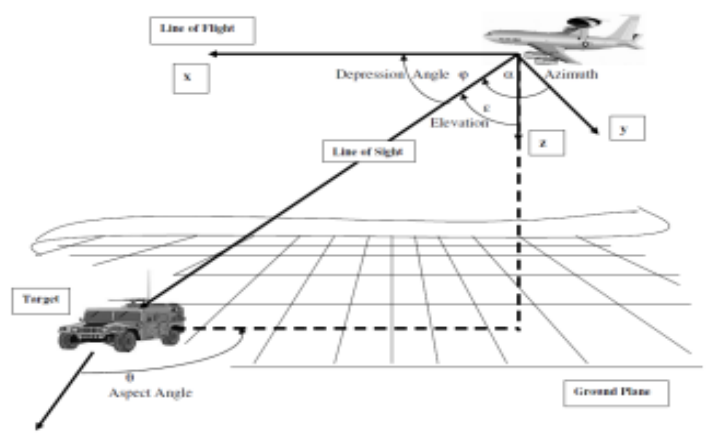

Figure 1 Target and sensor platform geometry

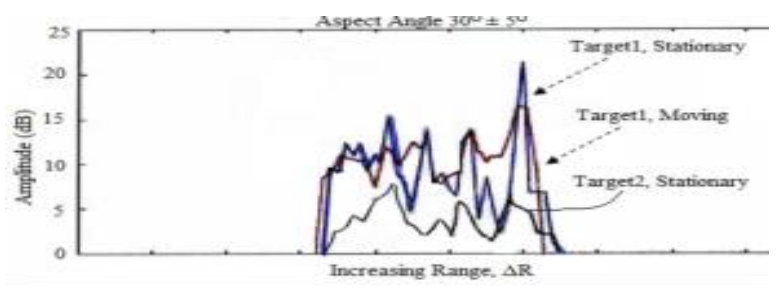

Figure 2 Continuous SAR range data over $\Delta \mathrm{R}$

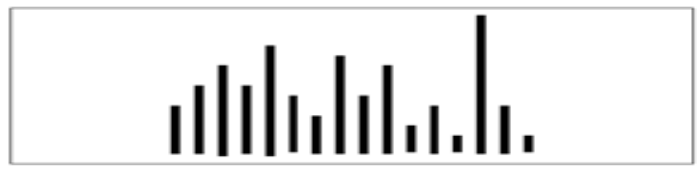

Figure 3 Digitized data, stationary target 1

level of 32 and 64 data points, and we also defined a synthetic target as a reference in statistical testing. In this paper we employ more precise data to make better target identification and pose estimate along the way. Figure 4 shows various components of our analysis, and Figures 5, 6, and 7 show three real life target signatures. Data resolution is $1 \mathrm{x} 1$ foot, depression angle is $15^{\circ}$. Figure 8 is our own made synthetic target used as a reference in our analysis.

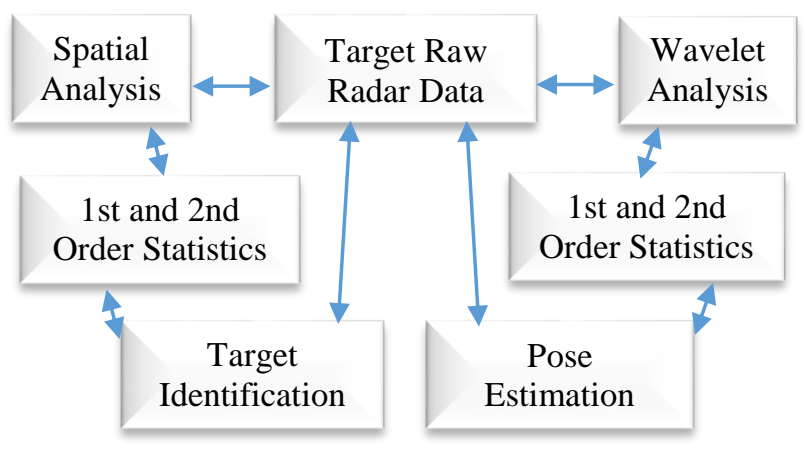

Figure 4. Target Identification and Pose Estimation

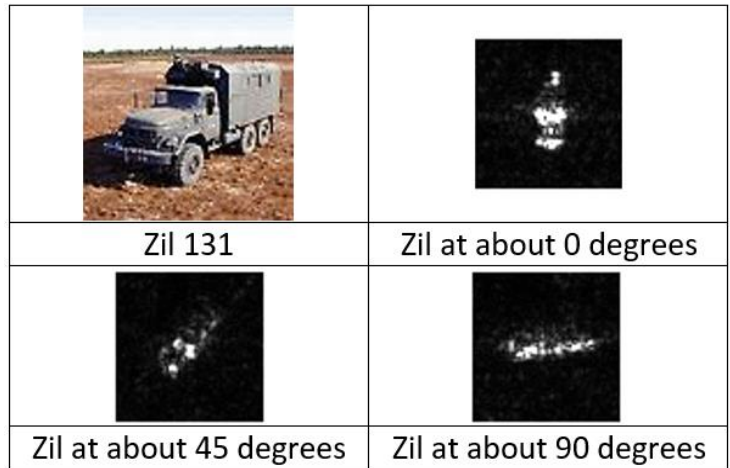

Figure 5. Zil truck raw SAR images

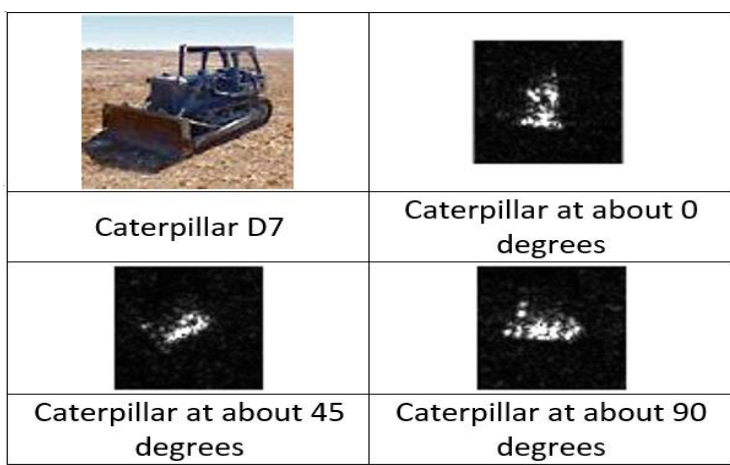

Figure 6. Caterpillar bulldozer raw SAR images

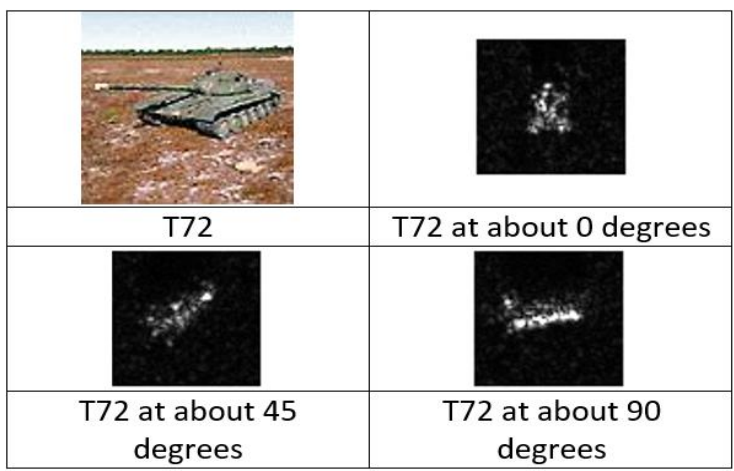

Figure 7. Tank T72 raw SAR images

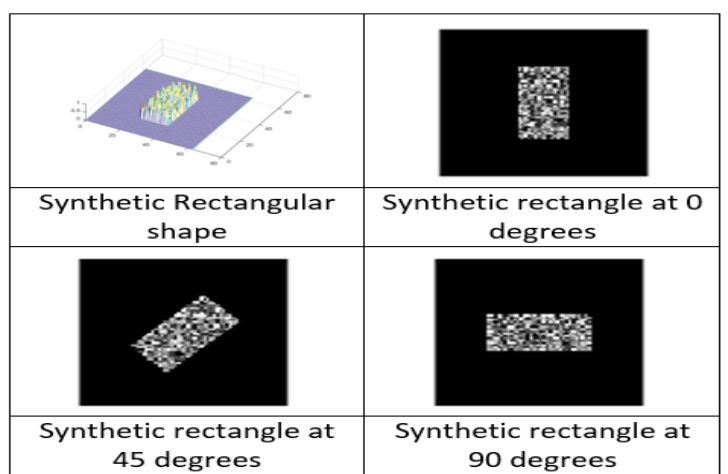

Figure 8. rectangular synthetic target with random pixels 


\section{TARGET SIGNATURES AS STOCHASTIC PROCESSES}

There are a total of 274 signatures for 4 quadrants, i.e. for $360^{\circ}$. These three real life targets span the range of different features and sizes. The MSAR data base [15-18], has more targets and our methodology can be applied to any of them, or to similar target data base. In order to analyze target signatures, let us first define two families of stochastic processes $\mathrm{X}(\mathrm{i}, \mathrm{j})=\mathrm{X}\left(\mathrm{T}_{\mathrm{i}}, \theta_{\mathrm{i}}\right)$ and $\mathrm{Y}(\mathrm{i}, \mathrm{j})=\mathrm{Y}\left(\mathrm{T}_{\mathrm{i}}, \theta_{\mathrm{i}}\right)$ as follows:

$$
\begin{aligned}
& X\left(T_{i}, \theta_{i}\right)=\text { Radar } X \text { signature, } i^{\text {th }} \text { target, } j^{\text {th }} \text { pose } \\
& Y\left(T_{i}, \theta_{i}\right)=\text { Radar } Y \text { signature, } i^{\text {th }} \text { target, } j^{\text {th }} \text { pose }
\end{aligned}
$$

where $\mathrm{I}=1,2,3,4$ (1-3 are real targets while 4 is synthetic), $\mathrm{j}=$ $1,2 \ldots 274\left(4\right.$ quadrants, $\left.360^{\circ}\right)$. Table 1 summarizes all target and pose combinations [5]. The vectors $X(I, j)$ and $Y(I, j)$ are $K$ dimensional; $X(I, j)=\left[X_{1}(I, j), X_{2}(I, j), \ldots, X_{k}(I, j)\right]^{T}, K$ is the number of radar signature pixels along $X$ and $Y$. Figure 3 shows $X(I, j)$ with $K=16$. In this paper $K=32$ or $K=64$ and similarly for $\mathrm{Y}(\mathrm{I}, \mathrm{j})$. This number is based on two considerations; first, the number of significant signature pixels for all targets is $\mathrm{K}$ or less. If less than $\mathrm{K}$, we append required number of zeros to the right of the signature. Second, for future research in wavelet domain, we want this number to be a power of 2. Signature numerical values are means along $X$ and $Y, X(I, j)$ and $Y(I, j)$, of pixels gray values from MSTAR data base. Figure 9 shows one Zil truck (Target 3) signatures, the gray scale levels are translated into digital signatures by averaging the gray level values along the $\mathrm{X}$ and $\mathrm{Y}$ axis. In other words if we take the average of pixels of Figure 5 when the truck is at zero degrees in both $\mathrm{X}$ and $\mathrm{Y}$ axis we get Figure 9. Figure 10 adds variances as well, which are useful in target and pose discrimination. Note that the signatures are filtered and very small values to the left and to the right are set to zeros. The last row in Table 1 indicates $j^{\text {th }}$ Pose Sample Statistics (PjSS) for any target, across $\mathrm{X}_{\mathrm{k}}(\mathrm{j})$ and $\mathrm{Y}_{\mathrm{k}}(\mathrm{j})$, with $\mathrm{k}=1,2, \ldots, \mathrm{K}, \mathrm{j}=1,2, \ldots, 274$. We dropped target indicator " $\mathrm{i}$ " for simplicity. These statistics are summarized in Table 2 for $\mathrm{X}(\mathrm{j})$, with similar table for Y(j). More on this in Section IV.

Table 1. Target Signature Template

\begin{tabular}{|c|c|c|c|c|c|}
\hline $\begin{array}{c}\text { Pose/ } \\
\text { Target }\end{array}$ & Pose 1 & Pose 2 & $\cdots$ & Pose 274 & $\begin{array}{c}\text { Signature/ } \\
\text { Correlation }\end{array}$ \\
\hline $\begin{array}{c}\text { Target1/ } \\
\text { Tank }\end{array}$ & $\begin{array}{c}X(1,1) / \\
Y(1,1)\end{array}$ & $\begin{array}{c}X(1,2) / \\
Y(1,2)\end{array}$ & $\cdots$ & $\begin{array}{c}X(1,274) / \\
Y(1,274)\end{array}$ & $\mathrm{T}_{1} \mathrm{SAC}$ \\
\hline $\begin{array}{c}\text { Target2/ } \\
\text { Bulldozer }\end{array}$ & $\begin{array}{c}X(2,1) / \\
Y(2,1)\end{array}$ & $\begin{array}{c}X(2,2) / \\
Y(2,2)\end{array}$ & $\cdots$ & $\begin{array}{c}X(2,274) / \\
Y(2,274)\end{array}$ & $\mathrm{T}_{2} \mathrm{SAC}$ \\
\hline $\begin{array}{c}\text { Target3/ } \\
\text { Truck }\end{array}$ & $\begin{array}{c}X(3,1) / \\
Y(3,1)\end{array}$ & $\begin{array}{c}X(3,2) / \\
Y(3,2)\end{array}$ & $\cdots$ & $\begin{array}{c}X(3,274) / \\
Y(3,274)\end{array}$ & $\mathrm{T}_{3} \mathrm{SAC}$ \\
\hline $\begin{array}{c}\text { Target4/ } \\
\text { Rectangle }\end{array}$ & $\begin{array}{c}X(4,1) / \\
Y(5,1)\end{array}$ & $\begin{array}{c}X(4,2) / \\
Y(4,2)\end{array}$ & $\cdots$ & $\begin{array}{c}X(4,274) / \\
Y(4,274)\end{array}$ & $\mathrm{T}_{4} \mathrm{SAC}$ \\
\hline Statistics & $\mathrm{P}_{1} \mathrm{SS}$ & $\mathrm{P}_{2} \mathrm{SS}$ & $\cdots$ & $\mathrm{P}_{274} \mathrm{SS}$ & $\mathrm{CTC}$ \\
\hline
\end{tabular}

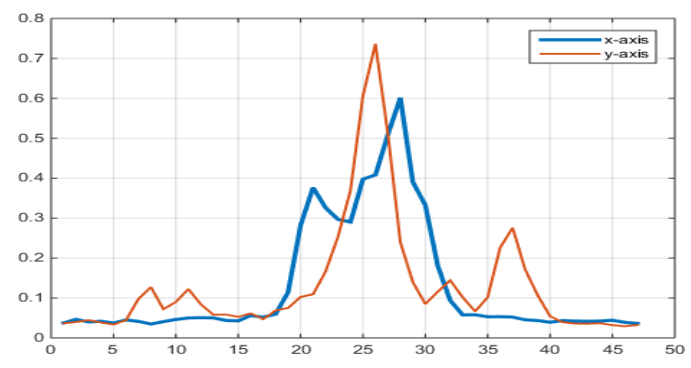

Figure 9. Raw to $\mathrm{X}$ and $\mathrm{Y}$ digital target signature

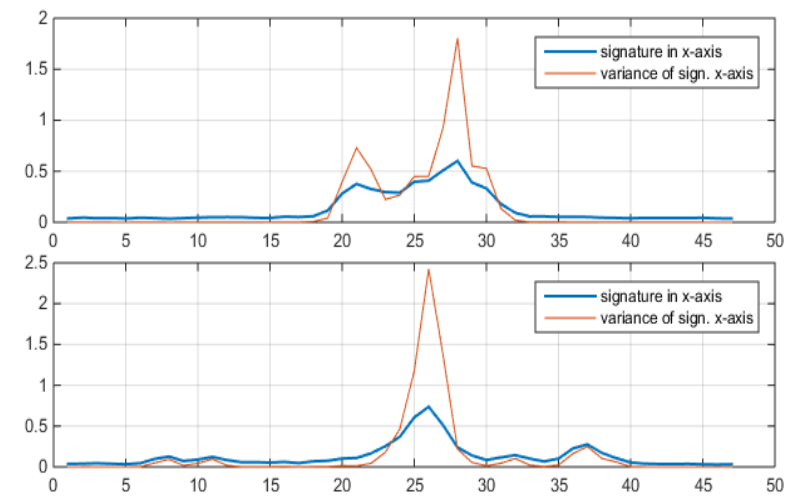

Figure 10. Target $\mathrm{X}$ and $\mathrm{Y}$ with corresponding variances

\section{TARGET GEOMETRY ANALYSIS}

In this Section we consider a simplified target geometry and assume we have a rectangular target. Figure 11 illustrates this idea in $(X, Y)$ coordinates using an angled target signature, between $0^{\circ}$ and $90^{\circ}$. Note that not every target will be a perfect rectangular shape but this is very good approximation for asymmetric target if we are interested in its maximum (length) and minimum (width) dimensions. From Figure 11 we easily obtain the following set of three nonlinear algebraic equations which summarize geometric relationships among three key geometric features of the target signature, i.e. width, lengths and pose angle. These equations need to be solved for X-s given $\mathrm{Y}$ s, when we acquire "real time" target as well as for justification of their use for the known data base targets.

$$
\begin{gathered}
\mathrm{X}_{1} \cos \left(\mathrm{X}_{3}\right)+\mathrm{X}_{2} \sin \left(\mathrm{X}_{3}\right)=\mathrm{Y}_{1} \\
\mathrm{X}_{1} \operatorname{sins}\left(\mathrm{X}_{3}\right)+\mathrm{X}_{2} \cos \left(\mathrm{X}_{3}\right)=\mathrm{Y}_{2} \\
\mathrm{X}_{1} \mathrm{X}_{2}=\mathrm{Y}_{3}
\end{gathered}
$$
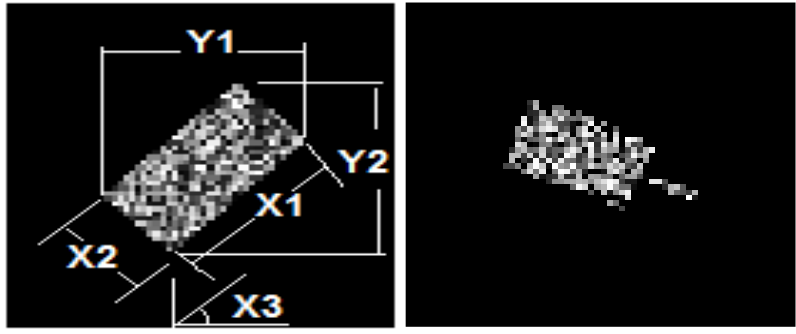

Figure 11. Geometry of ideal rectangular target (Left) Figure 12. Synthetic target pose determination (Right)

In order to calculate $Y_{1}$ and $Y_{2}$ we need to perform further analysis of the target signatures. Namely, we will need to "clean up" or "pre filter" target signatures by eliminating all zero or near zero amplitudes right before the target signature exhibits significant spatial amplitude, starting from the left side. We can call these signature leading zeroes. Similarly we will clean the trailing zero on the right side of the signature. Equations 2 can be solved for a "real time" target once acquired by the sensor platform. The solutions $X_{1}$ and $X_{2}$ correspond to the length and width of the rectangular target, and $X_{3}$ is corresponding pose angle estimate. The initial solution is generically presented in $1^{\text {st }}$ quadrant ( 0 to 90 degrees) but real quadrant is determined in the 
second step when we calculate $1^{\text {st }}$ order statistics. This will act as an initial estimate of pose and will identify the target type as well. Further use of other statistics will improve the estimate. That is the basic idea in our approach. The following example illustrates the approach. We chose a random synthetic target at $224^{\text {th }}$ position in the synthetic target data base (Figure 12 for simulated raw pixels, Figure 13 for averaged $\mathrm{X}$ and $\mathrm{Y}$ signatures). After solving the equations for $X_{1}, X_{2}$, and $X_{3}$ we get the first estimate of the target geometry and pose (Table 2). This information determines the target position at $50\left(1^{\text {st }}\right.$ quadrant), out of 274 in the circle. The real quadrant (4 in this case) is determined by analyzing three more signatures shifted 90 degrees from target 50, and situated in quadrants 2, 3 and 4 (Figure 14). Next we take three synthetic targets from target data base plus a number of additional signatures with poses around signature 50, shift them 90 degrees as well, and compare their signatures with the signatures in Figure 13. For these comparisons we use several correlation coefficients, namely Pearson, Spearman, Kendal, and also Brownian to identify the target and also right quadrant. Tables 3 and 4 show correlations for $\mathrm{X}$ and $\mathrm{Y}$, for these four correlation coefficients, for various poses, and their sum (boldfaced) as an ultimate measure of coincidence between the correlations. The correlations are at the peak with Target 1 and pose 225 (7.6881 vs 6.7915 and 6.4929), and the target is in $4^{\text {th }}$ quadrant. Hence we missed by one pose position, error of 1 in 274 or $0.365 \%$, which is an excellent estimate between (i) geometrical and (ii) correlation comparisons. See our papers [1,2] for more information on correlation coefficients. In general, this still does not mean we found the correct pose, it is an initial estimate only. More statistical tests can be done to find the correct pose.

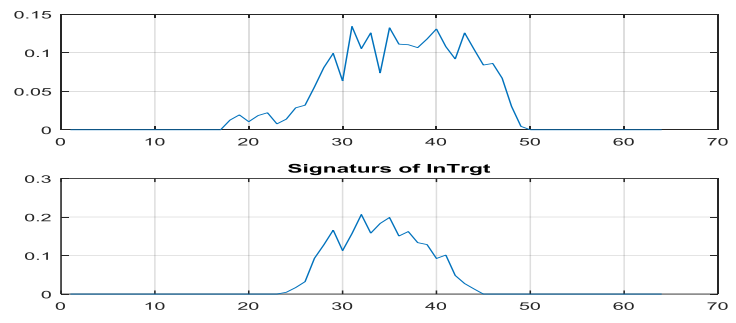

Figure 13. $\mathrm{X}$ and $\mathrm{Y}$ signatures for synthetic target

Table 2. Target Pose Estimation Example

\begin{tabular}{|c|c|}
\hline $\mathrm{X}_{1}$ (Width) & 8.98 pixels \\
\hline $\mathrm{X}_{2}$ (Length) & 31.06 pixels \\
\hline $\mathrm{X}_{3}$ (Pose) & 65.63 degrees \\
\hline Position No & 50 \\
\hline
\end{tabular}

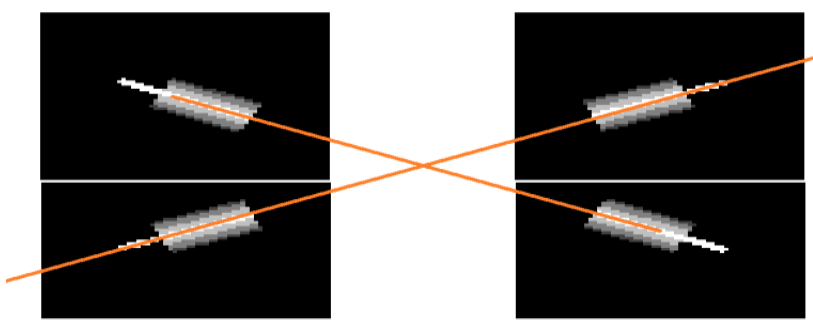

Figure 14. Geometric symmetricity of target signatures
Table 3. $1^{\text {st }}$ Target Correlations

\begin{tabular}{|c|c|c|c|c|}
\hline 223 & 224 & 225 & 226 & 227 \\
\hline 0,9697 & 0,9712 & 0,9719 & 0,9680 & 0,9724 \\
\hline 0,9751 & 0,9789 & 0,9795 & 0,9807 & 0,9809 \\
\hline 0,9762 & 0,9783 & 0,9824 & 0,9799 & 0,9789 \\
\hline 0,9462 & 0,9808 & 0,9803 & 0,9803 & 0,9645 \\
\hline 0,9004 & 0,9086 & 0,9213 & 0,9109 & 0,9073 \\
\hline 0,9001 & 0,9447 & 0,9419 & 0,9415 & 0,9231 \\
\hline 0,9395 & 0,9416 & 0,9425 & 0,9346 & 0,9444 \\
\hline 0,9624 & 0,9677 & 0,9682 & 0,9698 & 0,9701 \\
\hline 7,5696 & 7,6719 & $\mathbf{7 , 6 8 8 1}$ & 7,6657 & 7,6417 \\
\hline
\end{tabular}

Table 4. $2^{\text {nd }}$ and $3^{\text {rd }}$ Target Correlations

\begin{tabular}{|c|c|c|}
\hline 226 & 227 & 228 \\
\hline 0,8148 & 0,8212 & 0,8152 \\
\hline 0,9415 & 0,9476 & 0,9514 \\
\hline 0,8458 & 0,8496 & 0,8503 \\
\hline 0,9210 & 0,9213 & 0,9213 \\
\hline 0,7391 & 0,7507 & 0,7546 \\
\hline 0,8699 & 0,8694 & 0,8670 \\
\hline 0,7003 & 0,7095 & 0,7013 \\
\hline 0,9144 & 0,9224 & 0,9278 \\
\hline 6,7469 & $\mathbf{6 , 7 9 1 5}$ & 6,7890 \\
\hline
\end{tabular}$\quad$\begin{tabular}{|c|c|c|}
\hline 227 & 228 \\
\hline 0,7736 & 0,7761 \\
\hline 0,7227 & 0,9272 \\
\hline 0,9041 & 0,979 \\
\hline 0,6953 & 0,6911 \\
\hline 0,8474 & 0,8494 \\
\hline 0,6477 & 0,6508 \\
\hline 0,8892 & 0,8956 \\
\hline 6,4780 & $\mathbf{6 , 4 9 2 9}$ \\
\hline
\end{tabular}

We check the result (pose 225) by comparing X and Y signature variances. Without going into the details, we obtain the same pose 225 estimate. At this point we can continue with more statistical tests (Sections IV and V) to fine tune the estimate to correct pose 224 or be satisfied with estimate at 225 . For the real signatures there may be some additional pose estimation errors hence more tests could be required. See next Sections.

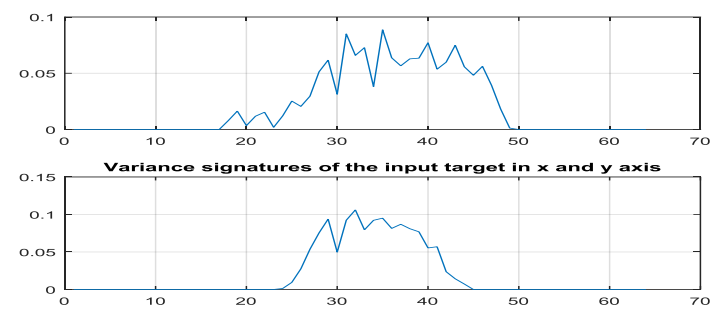

Figure 15. Target signature variances

\section{SIGNATURE STATISTICS ACROSS POSES}

Table 5 summarizes a list of signature statistics which can be used for further pose estimation refinement. We are currently looking into the importance of all of the individual statistics in order to form their optimal combination, for not all carry useful information. Table 5 is a summary of all the statistics, Figure 16 and 17 have their values for Zil truck (Figure 5) and ideal rectangular (Figure 8). Table 6 summarizes sample autocorrelations which are relevant for target identification and pose estimation. The autocorrelation measures signature correlation between components in $\mathrm{X}(\mathrm{i}, \mathrm{j})$, for each target and for every pose individually, for a total of 4 times 274, i.e. 1096 autocorrelations. Likewise for $\mathrm{Y}$ axes. The autocorrelation captures certain geometrical features of the targets. In particularly the zero crossing $\mathrm{X}_{\mathrm{zcor}}$ indicates the relative size of the signature projection onto $\mathrm{X}$. The zero crossing also corresponds to the number of non-zero signature samples. 
Table 5. List of Target Signature Statistics

\begin{tabular}{|c|l|}
\hline Statistics & \multicolumn{1}{c|}{ Description } \\
\hline$X_{\text {max }}, k_{\max }$ & Maximum signature and position among $k$ \\
\hline$X_{m}$ & Mean signature value $\Sigma_{k} X_{k}(j) / k$ \\
\hline$X_{\text {mead }}$ & Signature median, ordered middle value \\
\hline$X_{\text {var }}$ & Signature variance $\Sigma_{k}\left[X_{k}(j)-S_{\text {mean }}\right]^{2} / k$ \\
\hline$X_{\text {stedv }}$ & Signature standard deviation $\left(S_{\text {var }}\right)^{0.5}$ \\
\hline$X_{\text {vare }}$ & Signature variance energy $\Sigma_{k}\left[X_{k}(j)-S_{\text {mean }}\right]^{2}$ \\
\hline$X_{\text {max } / \text { mean }}$ & Signature max/mean ratio $S_{\text {max }} / S_{\text {mean }}$ \\
\hline$X_{\text {varm }}, k_{m v}$ & Max variance and position in $\mathrm{K}$ samples \\
\hline$X_{\text {energy }}$ & Signature energy $\Sigma_{k} X^{2}{ }_{k}(j)$ \\
\hline$X_{\text {skew }}$ & Signature skew Adjusted FP \\
\hline$X_{\text {skew }}^{I}$ & Adjusted FP signature skew \\
\hline$X_{\text {skewv }}^{l}$ & Adjusted FP signature skew variance \\
\hline &
\end{tabular}

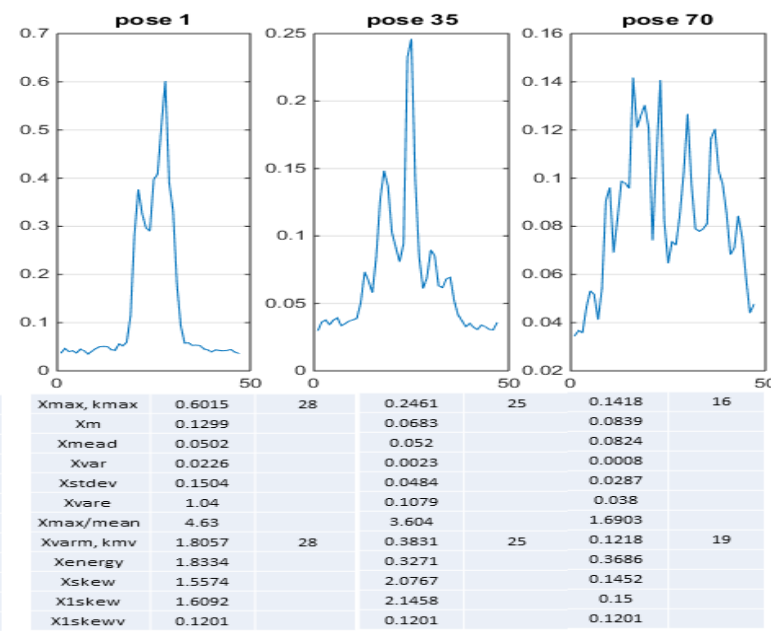

Figure 16. Zil truck signature statistics across 3 poses

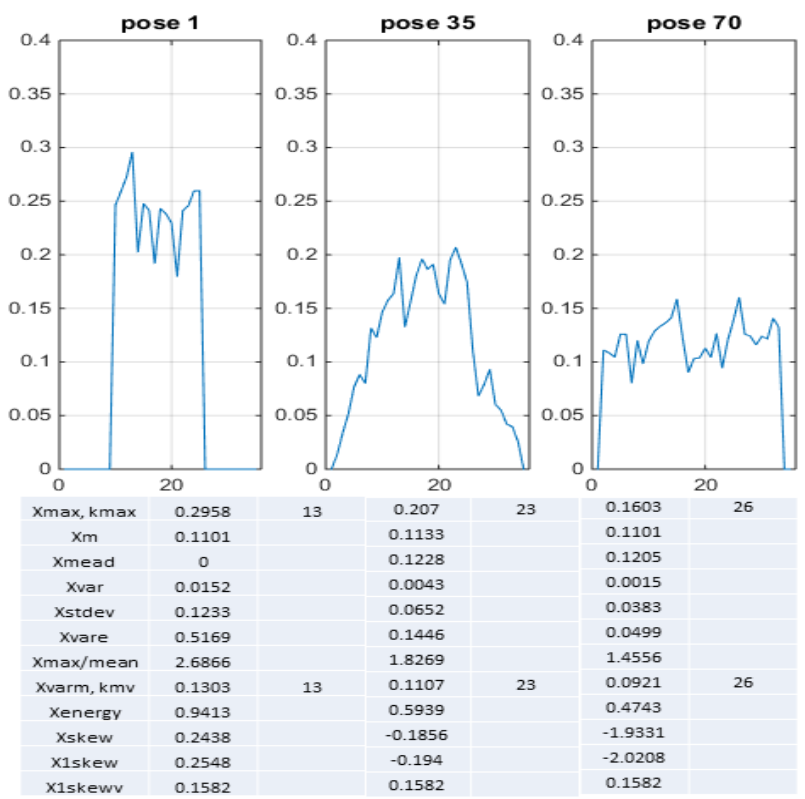

Figure 17. Synthetic signature statistics across 3 poses
Table 6. Pose j Sample Autocorrelations ( $\left.\mathrm{P}_{\mathrm{j}} \mathrm{SS}\right)$

\begin{tabular}{|c|l|}
\hline Variable & \multicolumn{1}{c|}{ Description } \\
\hline$X_{\text {cor }}$ & Signature autocorrelation function \\
\hline$X_{\text {zcor }}$ & Signature autocorrelation zero crossing \\
\hline$X_{\text {ecor }}$ & Signature autocorrelation error \\
\hline$X_{\text {npix }}$ & Number of pixels along X \\
\hline
\end{tabular}

We assume that signature has no leading zeros for they would shift autocorrelation zero crossing. As pose angle change we get different zero crossings for different target projection onto $\mathrm{X}$ and Y. In Section $\mathrm{V}$ we refer to zero crossing $\mathrm{man} / \mathrm{min}$ ratio, $\mathrm{Xz} / \mathrm{max} / \mathrm{min}$, which indicates approximate length/width ratio. Zil truck dimensions can be found on line and compared. Figure 17 shows ideal rectangular target, with length $\mathrm{L}=32$, and width $\mathrm{W}=16$ feet, for 1 foot resolution as in MSTAR. Here zero crossings correspond to $\mathrm{W}, \mathrm{L}$ and $45^{\circ} \mathrm{X}$ projection, so that we have $\mathrm{X}_{\text {proj }}=\mathrm{Lsin}(45)+\mathrm{W} \cos (45)=35.6$.

\section{SIGNATURE STATISTICS ACROSS ONE TARGET}

The last column in Table 1 indicates $i^{\text {th }}$ Target Signature Auto Correlation $\left(\mathrm{T}_{\mathrm{i}} \mathrm{SAC}\right)$ features for each of four targets across every pose, for both $\mathrm{X}(\mathrm{i}, \mathrm{j})$ and $\mathrm{Y}(\mathrm{i}, \mathrm{j})$. We first consider two correlation measures, Pearson and Spearman coefficients. Then we look into pose shifted autocorrelations. Table 7 has corresponding statistics summary.

Table 7. Auto Correlations Features ( $\left.\mathrm{T}_{\mathrm{i}} \mathrm{SAC}\right)$

\begin{tabular}{|c|l|}
\hline Variable & \multicolumn{1}{|c|}{ Description } \\
\hline$X_{\mathrm{p}}$ & Pearson coefficient \\
\hline$X_{\mathrm{s}}$ & Spearman coefficient \\
\hline $\mathrm{X}_{\text {psauto }}$ & Pose shifted autocorrelation envelope \\
\hline $\mathrm{X}_{\text {zpauto }}$ & Pose shifted autocorrelation zero crossing \\
\hline $\mathrm{X}_{\mathrm{z} / \mathrm{max} / \min }$ & Autocorrelation max/min zero crossing \\
\hline
\end{tabular}

We can start with $X(1,1)$ and correlate it with $X(1,2)$, then with $X(1,3)$, etc., and finally with $X(1,274)$ forming envelope of correlations. This results in a $4 \times 274=1096$ correlations. These capture cross statistics for poses and can be used to discriminate poses. Figure 18 and 19 show Pearson and Spearman coefficients, as well as correlation envelopes, as a function of the pose for Zil Truck and for our rectangular synthetic target.
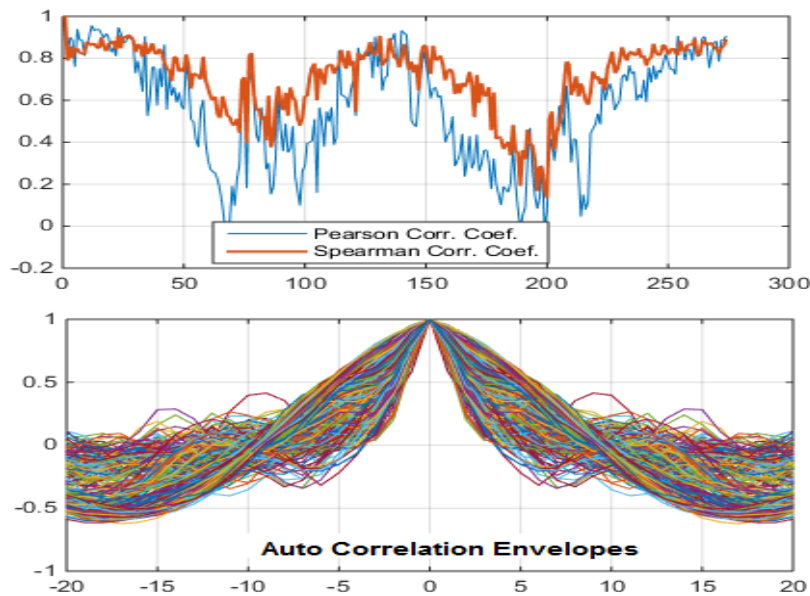

Figure 18. Zil truck pose shifted correlations 


\section{STATISTICS ACROSS DIFFERENT TARGETS}

The final step in our target signature analysis is to look at the cross target statistics and correlations. We will make a practical assumption that the corresponding target signatures form sets of independent stochastic processes, or at least uncorrelated (orthogonal) processes. For example we correlate $\mathrm{X}(1, \mathrm{j})$ with $X(2, j)$, then with $X(3, j)$, and with $X(4, j)$, assuming the same pose angle $\mathrm{j}$, against independence or uncorrelated (orthogonal) assumption. Each of correlations produces an envelope for 274 poses, for total of 4 envelopes. In view of that, we use cross covariance and correlations to test lack of correlation, and also classic Chi-Square and newly introduced Brownian Distance Covariance [21], to test for independence among various target signatures. Also, when a "real time" target signature is acquired, it will also be tested against all other four sets of stored target signatures. Table 8 summarizes target cross correlation features, both for $\mathrm{X}$ and $\mathrm{Y}$ signatures.

Table 8. Cross Targets Correlation Features (CTC)

\begin{tabular}{|c|l|}
\hline Variable & \multicolumn{1}{c|}{ Description } \\
\hline$\chi$ & Cross target Chi-Square test \\
\hline $\mathrm{V}^{2} \mathrm{k}(\mathrm{i}, \mathrm{j})$ & Cross target Brownian distance covariance \\
\hline $\mathrm{R}^{2} \mathrm{k}(\mathrm{i}, \mathrm{j})$ & Cross target Brownian distance correlation \\
\hline $\mathrm{V}^{2} \mathrm{k}$ & Target Brownian sample distance variance \\
\hline $\mathrm{X}_{\mathrm{ccor}}$ & Cross target correlations \\
\hline
\end{tabular}

Chi square test is used to test the independency of two sequences $[1,2]$. The test returns the value from Chi-squared distribution and the degrees of freedom number [22-24]. The test is defined as: Where $A_{k}=$ actual sample value, and $E_{k}=$ expected sample value, and the sum is from 1 to $K$. We choose $A_{k}=$ $\mathrm{X}_{\mathrm{k}}(1, \mathrm{j})$ and vary $\mathrm{E}_{\mathrm{k}}$ so that $\mathrm{E}_{\mathrm{k}}=\mathrm{X}_{\mathrm{k}}(2, \mathrm{j}), \mathrm{E}_{\mathrm{k}}=\mathrm{X}_{\mathrm{k}}(3, \mathrm{j})$, and $\mathrm{E}_{\mathrm{k}}=$ $X_{k}(4, j)$. Similarly if a "real target" is chosen. Low values of $\chi^{2}$ is an indicator of independence. The value of $\chi^{2}$ is always positive or 0 if $A_{k}=E_{k}$ for every k. See Figure 20 .

Once $\chi^{2}$ is calculated, a program like Matlab or Excel, returns "the probability $P$ that a value of $\chi^{2}$ statistic at least as high as the value calculated could happen by chance under the assumption of independence", with an appropriate number of degrees of freedom, $\mathrm{df}=\mathrm{K}-1$. The test is most appropriate when $\mathrm{E}_{\mathrm{k}}$ 's are $\geq 5$, hence we scale the signatures for that condition.
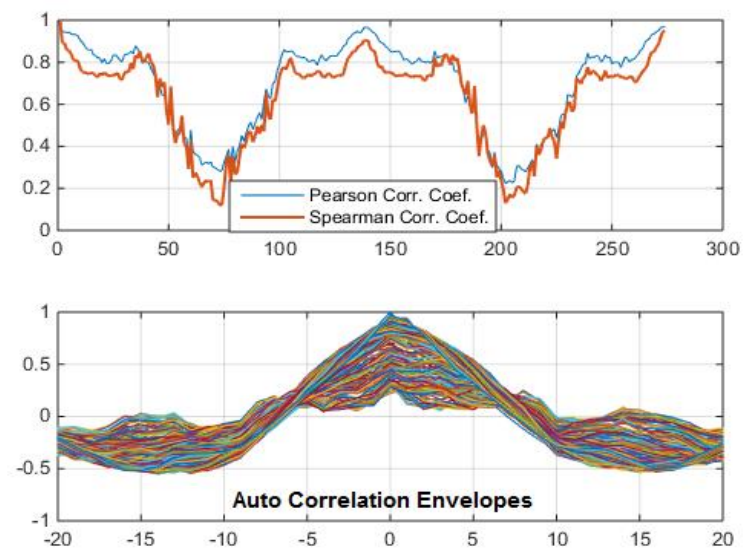

Figure 19. Ideal rectangular pose shifted correlations
Finally, we use the newest form of correlation [21], i.e. distance covariance and correlation (Brownian distance covariance and correlation). The key is that zero correlation implies independence, plus the method captures non stationary and nonlinear correlations as well.

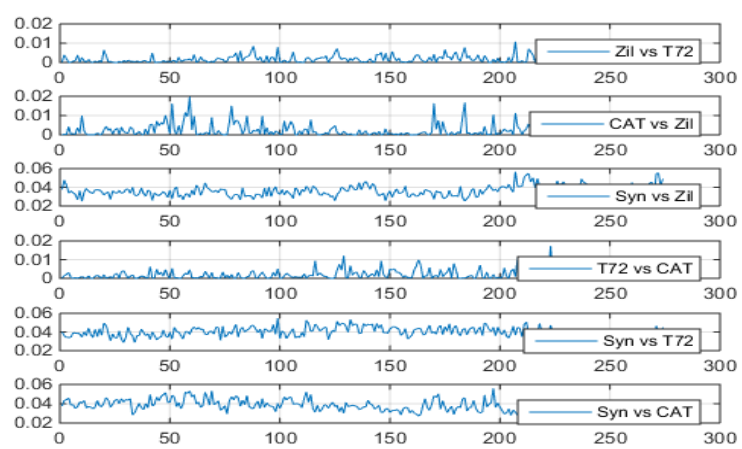

Figure 20. Cross target chi-square test

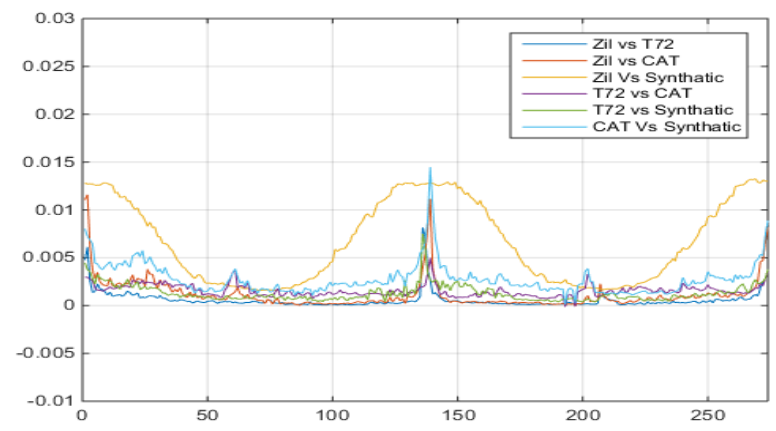

Figure 21. Cross target Brownian distance covariance

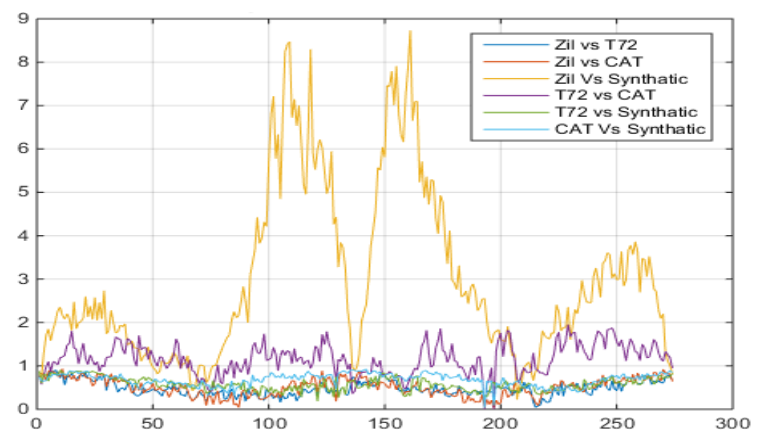

Figure 22. Cross target Brownian distance correlation

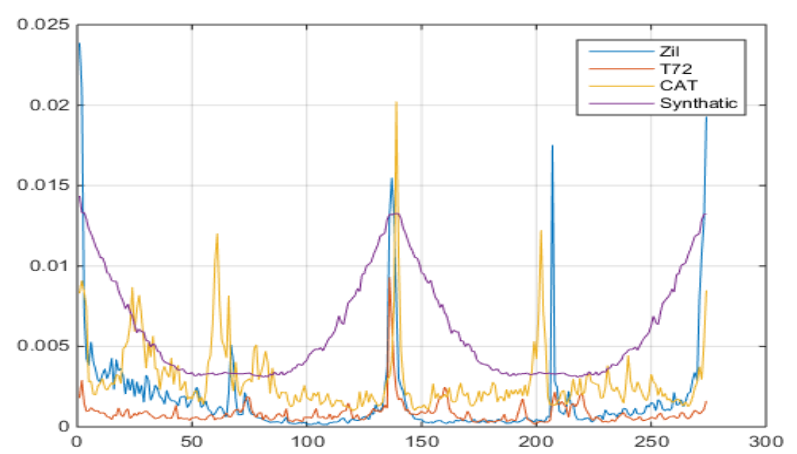

Figure 23. Target Brownian sample distance variance 


\section{CONCLUSION}

In this paper we present continuation of our previous work in analysis of digital target signatures and the corresponding problem of pose estimation. Quality pose estimation is a required step in subsequent automatic target tracking operation. The target signatures are obtained from USA MSTAR public data base where variety of commercial as well as military targets are included, obtained by SAR and HRR radars. The methodology we employ is based on geometrical as well as several statistical correlation measures such as various autocorrelations and cross-correlations, as well as Brownian and Chi Square statistical tests for sequence independence, which compare stored target data base against real time target. In particular we start target identification and pose estimation process by (i) using target geometry which reliably determines the target type and $1^{\text {st }}$ quadrant pose estimate, we then follow with (ii) variety of first order statistics to further fine tune the pose, and (iii) more $2^{\text {nd }}$ order statistics for the final pose refinement. In this paper we presented first two items for synthetic targets, and are currently working on the item (iii) and real target signatures. Certain geometrical features of the targets can be identified from the corresponding correlations. In a follow up research we will address all these additional items as well as corresponding frequency based on Haar wavelet analysis, combine it with spatial analysis, and use hypothesis testing when determining real target identification and pose estimate. These input will serve as entry data for target tracking.

\section{References}

[1] M. Hodzic and T. Namas, "Pose estimation methodology for target identification and tracking", in Proceedings of International Conference on Systems, Control, Signal Proceesing \& Informatics, INASE SCSI, ECS April 2015.

[2] M. Hodzic and T. Namas, "Spatial Analysis for Target Signatures ", IEEE ICAT Proceedings, XXV International Conference on Information, Communication and Automation Technologies Sarajevo, October 2015.

[3] B. Kahler and E. Blasch, "Robust multi-look hrr atr investigation through decision - level fusion evaluation," in Proc. $11^{\text {th }}$ International Conference on Information Fusion 2008

[4] B. Kahler, J. Querns, “ An atr challenge problem using hrr data," in Proc. SPIE, Vol 6970, 2008.

[5] J. Layne and D. Simon, "A multiple model estimator for a tightly coupled HRR automatic target recognition and MTI tracking system", in SPIE Conference on Algorithms for Synthetic Aperture Radar Imagery, 1999..

[6] E. Blasch, "Derivation of a belief filter for high range resolution radar simultaneous target tracking and identification," Ph.D. dissertation, Wright State University, 1999.

[7] E. Blasch and C. Yang, "Ten methods to fuse gmti and hrrr measurements for joint tracking and id," in Fusion 04, 2004.

[8] D. Gross, M. Oppenheimer, B. Kahler, B. Keaffaber, and R. Williams, "Preliminary comparison of hrr signatures of moving and stationary ground vehicles," in Proc. SPIE, Vol. 4727, 2002.

[9] R. Williams, J. Westerkamp, D. Gross, and A. Palomino, "Automatic target recognition of time critical moving targets using 1D high range resolution (HRR) radar," IEEE AES Systems Magazine, 2000.
[10] R. Mitchell and J. Westerkamp, "Robust statistical feature based aircraft identification," IEEE Trans. Aerospace \& Electronic Systems, vol. 35, no. 3, 1999.

[11] E. Blasch, J. Westerkamp, J. Layne, L. Hong, F. D. Garber, and A. Shaw, "Identifying moving HRR signatures with an ATR belief filter", SPIE, 2000.

[12] E. Blasch and S. Huang, "Multilevel feature-based fuzzy fusion for target recognition", in SPIE, 2000.

[13] W. Snyder, G. Ettinger, and S. Laprise, "Modeling performance and image collection utility for multiple look ATR", in SPIE, 2003.

[14] F. Dicander and R. Jonsson, "Comparison of some hrr classification algorithms," in SPIE, 2001.

[15] JSTARS, Joint Surveillance and Target Attack Radar System USA, from The Website for Defense Industries, Air Force, December 2001.

[16] Joint STARS/JSTARS, Intelligence Resource Program, FAS Website, December 2001.

[17] Joint STARS in Bosnia, Too Much Data Too Little Intel, Military Intelligence Professional Bulletin, http//fas.org/irp/agency/army/tradoc/usaic/mipb/19964/agee.htm.

[18] MSTAR online databe www.sdms.afrl.af.mil

[19] G. J. Szekelyi and M. L. Rizzo, "Brownian distance covariance," The Annals of Applied Statistics, vol. 3, no. 4, 2009.

[20] A. Leon-Garcia, "Probability, Statistics, and Random Processes for Electrical Engineering", $3^{\text {rd }}$ Edition, Pearson Prentice Hall, 2008. 